\title{
Catalog of the emission lines in the visible spectrum of comet 153P/lkeya-Zhang ${ }^{\star}$
}

\author{
G. Cremonese ${ }^{1}$, M. T. Capria ${ }^{2}$, and M. C. De Sanctis² \\ 1 INAF - Osservatorio Astronomico, Vicolo Osservatorio 5, 35122 Padova, Italy \\ e-mail: cremonese@pd.astro.it \\ 2 INAF - IASF, Area di Ricerca Tor Vergata Rome, Italy \\ Received 7 October 2005 / Accepted 3 August 2006
}

ABSTRACT

\begin{abstract}
A program of high resolution spectroscopy of comets is being conducted at TNG (Canary Islands) using the echelle spectrograph SARG. The aim of the program is to catalogue emission lines in the visible range of various comets, compare them and possibly identify the many existing unknown lines. As a first step, we catalogued the lines in the spectrum of the comet 153P/Ikeya-Zhang and compared them with the lines already listed in existing catalogues. The comet was observed with SARG on the night 19-20 of April, 2002, with a short slit covering the spectral range of 4620-7920 ̊ and with a resolving power $R=57000$, finding 8468 emission lines.
\end{abstract}

Key words. line: identification - techniques: spectroscopic - catalogs - comets: individual: Ikeya-Zhang

\section{Introduction}

The study of comets is very important to understand the early evolution of the Solar System, because comets are thought to contain the matter least modified since the formation of our Solar System. Most of our knowledge about comets comes from the study of the coma, much more simple to observe than the nucleus. All the species that can be observed in the coma of a comet were once contained in the nucleus, and are called parent molecules, or are the product of a physical process that affected a parent molecule and are called daughter molecules. Parent molecules can be directly observed only by measuring the radiation emitted in the IR or radio wavelengths, while daughter molecules emit mainly in the visible and ultraviolet wavelengths.

The visible spectral range is crowded with lines, and between them many important lines of daughter molecules and ions are still to be identified. The study of these lines could provide a large amount of information on the composition and the chemical processes working in the coma, on the environment of molecular formation or condensation and of the thermal history of cometary ices: we still have to understand some key points on the origin and evolution of a comet related to chemical processes and composition and the analysis of molecular and atomic emission lines would help this. Low resolution visible spectra have been obtained for many comets and in several examples it would be very useful to obtain the identification of the lines in the region of interest with high resolution $(R>40000)$ spectra. Up to now very few comets have been observed at high spectral resolution, due to the few high resolution spectrographs available and the difficulty to obtain time at the telescopes when the comet is bright enough. Every time it has been possible to get high

* Catalog, full Table 3 and Tables 4-9 are only available in electronic form at the CDS via anonymous ftp to

cdsarc.u-strasbg.fr (130.79.128.5) or via

http://cdsweb.u-strasbg.fr/cgi-bin/qcat?]/A+A/461/789 resolution spectra of a comet much information has been obtained, depending on the wavelength range covered, but most of these observations have been concentrated on small spectral regions. The advent of echelle spectrographs gives us the possibility to obtain high resolution spectra in a large wavelength region.

A program of high resolution spectroscopy of comets has been started at the Telescopio Nazionale Galileo (TNG) on La Palma (Canary Islands), using the echelle spectrograph SARG; four comets have been observed (153P/Ikeya-Zhang, 1P/Encke, C/2001 Q4 (NEAT) and 9P/Tempel 1). Our first objective is to obtain a catalog of emission lines over a wide spectral range with a resolving power greater than 40000 for a large sample of comets, in order to make comparisons and to study the interesting lines. The sample of comets for which emission line catalogs exist is so far very poor, and only new and long period comets have been observed. Only three catalogues of this type exist (Brown et al. 1996; Zhang et al. 2001; Cochran \& Cochran 2002). The catalog by Cochran \& Cochran (2002) is by far the most complete, because identifications made using lists of lines measured in the laboratory, as done by Brown et al. (1996), but the resolution of the spectra is higher $(R=60000)$. In the catalog published by Zhang et al. (2001), reporting a short list of emission lines for comet Hale-Bopp, the line identifications were based only on wavelength coincidences with the Brown et al. (1996) catalog. For each observed comet thousands of emission lines have been measured and about $20 \%$ were unidentified. It is well known that in the visible range several unidentified lines exist (Arpigny 1995); while it is possible that minor unknown species are hidden between these lines, most of them probably originate from not-yet catalogued transition lines of well known species $\left(\mathrm{NH}_{2}, \mathrm{C}_{2}\right.$.) or isotopes. The identification of these lines is our long term aim; it is a very difficult task, due to the lack of suitable laboratory lists and the difficulty in using existing ones. It is also of interest to compare the unidentified lines found in the 

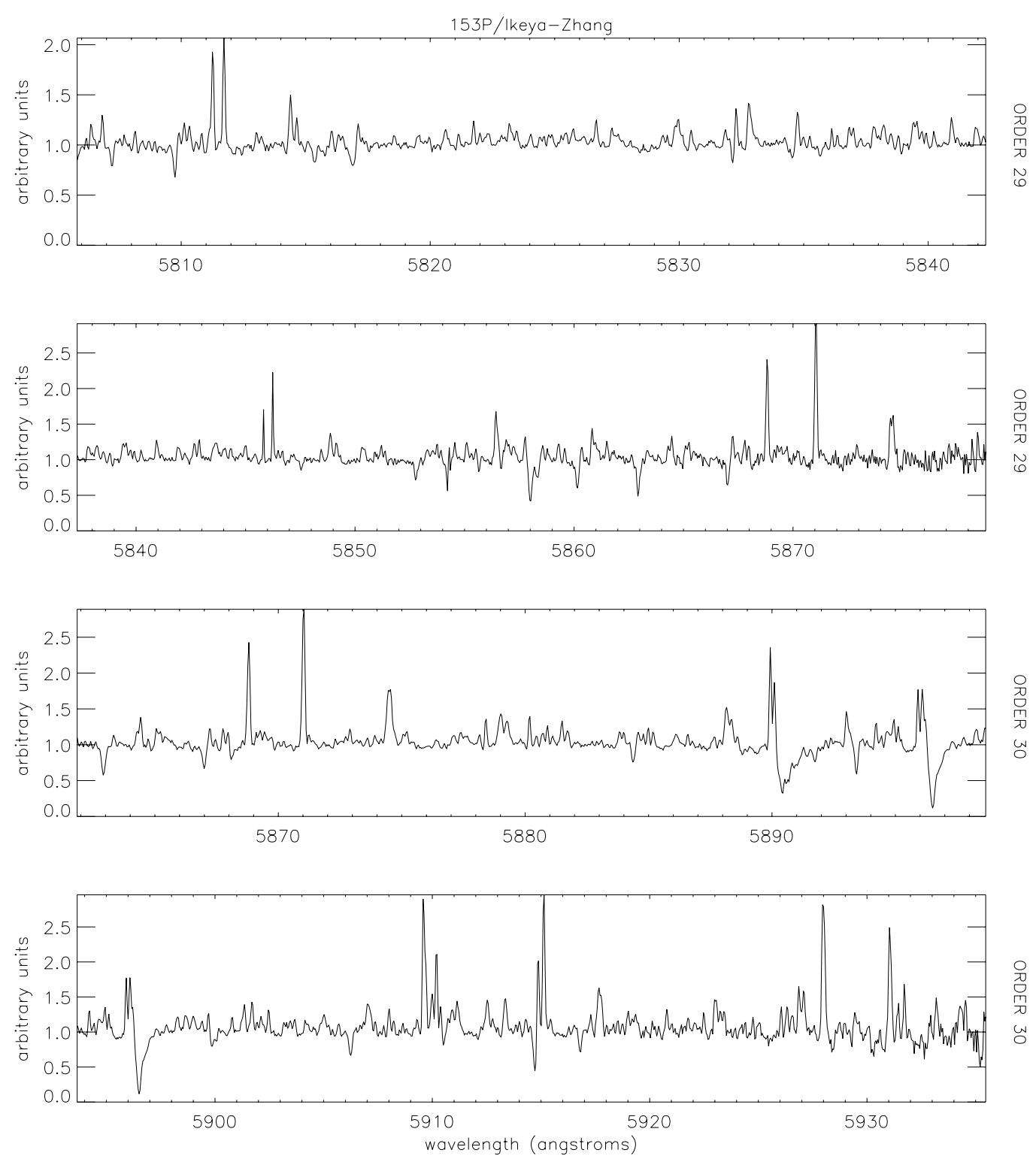

Fig. 1. Orders 29 and 30, in the $y$ axis we report the relative intensity in arbitrary units.

observed comets and analyze the differences: they may be due to a different origin and/or evolution, providing different composition and physical characteristics, or the different heliocentric and geocentric distances of observation, suggesting different thermodynamic environments.

\section{2. $153 \mathrm{P} / \mathrm{lkeya-Zhang}$}

The comet 153P/Ikeya-Zhang, discovered on February 2002 (Ikeya \& Zhang 2002), passed at perihelion on the 18th March 2002 and was faintly visible to the naked eye in March and April. This comet seems to be the return of a comet seen in 1661, C/1661 C1 (Marsden \& Nakano 2002).

Comet $153 \mathrm{P} /$ Ikeya-Zhang has been observed previously. Kawakita \& Watanabe (2002) reported that unidentified molecular bands, recognized in the plasma tail of several comets, are detected in the antisunward coma of the comet 153P/Ikeya-Zhang. They suggested that the unidentified band at $5310 \AA$ is correlated to the flux of $\mathrm{H}_{2} \mathrm{O}^{+}$.

The visible spectrum that is the subject of this paper (see the following section) has already been used for the
Table 1. Observing parameters.

\begin{tabular}{lc}
\hline \hline UT date & 20 Apr. 2002 \\
\hline$r(\mathrm{AU})$ & 0.89 \\
$\dot{r}\left(\mathrm{~km} \mathrm{~s}^{-1}\right)$ & 29.06 \\
$\Delta(\mathrm{AU})$ & 0.43 \\
$\dot{\Delta}\left(\mathrm{km} \mathrm{s}^{-1}\right)$ & -8.3 \\
Exposure time (s) & 2400 \\
\hline
\end{tabular}

determination of the nuclear spin temperature of ammonia (Kawakita et al. 2004) and of the ratio of atomic oxygen lines (Capria et al. 2005), confirming the usefulness of the high resolution spectrum of a comet.

$153 \mathrm{P} /$ Ikeya-Zhang is one of the few comets for which the nuclear spin temperature of ammonia has been estimated (Kawakita et al. 2004) and is about $32 \mathrm{~K}$. The result is similar to previous measurements in three other comets, C/2001 A2, C/1999 S4 and C/1995 O1: the nuclear spin temperatures of ammonia in these comets are around $30 \mathrm{~K}$ (Kawakita et al. 2004). These nuclear spin temperatures were measured under quite 
different conditions (heliocentric distances and gas production rates) and it would suggest that the spin temperature might reflect the physical temperature of dust grains where the molecules formed.

The atomic oxygen emission lines were the subject of a dedicated paper (Capria et al. 2005). The intensity ratio of the green to the red doublet was measured and the value found is compatible with the hypothesis that the lines originate mainly from the dissociation of the water molecule.

\section{Observations and data reduction}

Comet 153P/Ikeya-Zhang was observed on April 20 with the cross dispersed echelle spectrograph SARG on the $3.5 \mathrm{~m} \mathrm{TNG}$ telescope in La Palma (Canary Islands, Spain). At the moment of observation, the comet had a heliocentric distance of $0.89 \mathrm{AU}$ and a geocentric distance of $0.43 \mathrm{AU}$ (Table 1). A selection of 6 slits is available on the spectrograph: a slit can be selected working on a wheel at the main aperture. Each slit provides a different resolving power, to be selected according to the brightness of the target object. A slit of $0.8 \times 5.3$ arcsec was used, providing a resolving power $R=57000$, with a yellow grism allowing us to cover a spectral range of 4620-7920 $\AA$ and blazed at $5889 \AA$. Each order has been summed over the pixels along the full length of the slit.

The detector is a mosaic of $22 \mathrm{~K} \times 4 \mathrm{~K}$ thinned and back illuminated CCDs with a $13.5 \mu \mathrm{m}$ pixel size $(0.16 \mathrm{arcsec} / \mathrm{pixel})$, where the first chip includes the first 34 orders and the second one the following 21 . The complete set of plots showing all the orders are available in electronic form at the CDS and Fig. 1 reports the orders 29 and 30. On each plot the scale has been adjusted to the line of maximum intensity.

The data were reduced using the ECHELLE package of IRAF. Incandescent lamp observations were used to determine the flat field; ThAr lamp spectra were used to calculate the dispersion curve and, by fitting Thorium line positions for each order, a dispersion solution was achieved with rms errors lower than $7 \mathrm{~m} \AA$. The blaze function of each order has been corrected, fitting the continuum and dividing by the result of the fit. Each order has an overlapping region in the adjacent orders increasing toward the blue. Table 2 shows the wavelength range covered by each order.

\section{Emission line identification}

Emission features were searched for in the spectrum and the telluric emission lines were identified in the list of the measured lines. These telluric lines are due primarily to $\mathrm{OH}$ and $\mathrm{O}_{2}$, but $\mathrm{Na}$ and atomic oxygen lines are also present. In the range observed, the telluric lines are found both in emission and in absorption: while for the absorption lines the lost information cannot be recovered, the emission lines can be identified and removed using the catalog by Osterbrock et al. (1996). The lines in the catalog have been Doppler-shifted by the cometary geocentric velocity.

The identification of the remaining lines was based on the catalog and the context plots produced by Cochran \& Cochran (2002), available on the web. All the lines were identified in the above cited list and by inspection of the related plots.

Many orders have some overlap in the dispersion direction with the consecutive orders, and both order edges are often distorted. In order to treat with these problems, we adopted the following procedure: the distorted sections of each order
Table 2. Wavelength coverage of the different orders.

\begin{tabular}{cccccc}
\hline \hline Order & Range $(\AA)$ & Order & Range $(\AA)$ & Order & Range $(\AA)$ \\
\hline 1 & $4585-4640$ & 20 & $5340-5410$ & 38 & $6420-6500$ \\
2 & $4625-4675$ & 21 & $5390-5465$ & 39 & $6485-6570$ \\
3 & $4650-4710$ & 22 & $5440-5515$ & 40 & $6560-6640$ \\
4 & $4685-4745$ & 23 & $5490-5565$ & 41 & $6620-6715$ \\
5 & $4720-4785$ & 24 & $5530-5620$ & 42 & $6700-6785$ \\
6 & $4755-4820$ & 25 & $5590-5665$ & 43 & $6775-6865$ \\
7 & $4795-4860$ & 26 & $5640-5720$ & 44 & $6850-6940$ \\
8 & $4830-4900$ & 27 & $5695-5770$ & 45 & $6930-7020$ \\
9 & $4870-4940$ & 28 & $5745-5825$ & 46 & $7015-7100$ \\
10 & $4910-4980$ & 29 & $5800-5880$ & 47 & $7090-7180$ \\
11 & $4950-5020$ & 30 & $5860-5940$ & 48 & $7175-7260$ \\
12 & $4990-5660$ & 31 & $5915-6000$ & 49 & $7260-7360$ \\
13 & $5030-5100$ & 32 & $5975-6060$ & 50 & $7350-7440$ \\
14 & $5075-5145$ & 33 & $6035-6120$ & 51 & $7430-7540$ \\
15 & $5120-5190$ & 34 & $6095-6180$ & 52 & $7530-7625$ \\
16 & $5160-5230$ & 35 & $6235-6300$ & 53 & $7620-7720$ \\
17 & $5205-5280$ & 36 & $6280-6365$ & 54 & $7720-7820$ \\
18 & $5250-5320$ & 37 & $6350-6435$ & 55 & $7820-7920$ \\
19 & $5295-5370$ & & & & \\
\hline
\end{tabular}

were discarded, and the overlapping portions superimposed, inspected and compared with the Cochran \& Cochran (2002) plots. Emission lines were listed and included in our catalog only when they could be found, with similar intensity, in both the overlapping sections of an order.

The quality of the spectrum is comparable with the spectra of comet 122P/de Vico, published by Cochran \& Cochran (2002); the context is so similar that almost every line found in our spectrum, even the weakest, has a corresponding match in the spectrum of 122P/de Vico. In Fig. 2 we show a section of the order 39 with the labels identifying the emission.

The catalogs published by Brown et al. (1996) and Zhang et al. (2001) were used to cover the few gaps existing in the catalog published by Cochran \& Cochran (2002) but, due to the lack of accompanying plots and the lower wavelength resolution, these identifications should be considered less accurate: we listed in our tables only emission present in both catalogues.

In this way, we have obtained a list of 8468 lines, 1862 of which unidentified ("Unid" in the tables). In Table 3 we reproduce a small section of our catalog, corresponding to the lines shown in Fig. 2. In our list we have included many blended lines, noted with a "+" sign: we have assumed that, each time a line was clearly a blend of more lines, all the transitions listed in that position in the catalog by Cochran \& Cochran (2002) may be present. In the cited catalogs (Brown et al. 1996; Zhang et al. 2001) a similar approach was followed.

The complete table, containing all the emission lines found in our spectrum sorted by wavelength, is available in electronic form at the CDS. The Tables 4-9 containing the emission lines listed by species $\mathrm{C}_{2}, \mathrm{NH}_{2}, \mathrm{CN}, \mathrm{H}_{2} \mathrm{O}^{+}$, atomic species, and unidentified emissions respectively are also available at the CDS, with the same columns as Table 3 .

\section{Discussion and conclusion}

Most of the lines we have found are $\mathrm{C}_{2}, \mathrm{NH}_{2}$ and $\mathrm{CN}$ (respectively, 4723, 1572 and 216 lines for each individual table), but we also have found 89 lines attributed to $\mathrm{H}_{2} \mathrm{O}^{+}$. We have only one spectrum taken in the optocenter of the comet, so we cannot check this with a spectrum taken in the tail, as done by Cochran \& Cochran (2002), but we are confident about their attribution 
Table 3. From the table containing all the emission lines found in the spectrum, available at the CDS. The first column is the wavelength in angstrom; the second column the relative peak intensity; the third column contains the molecule name (or "Unid" when the line is unidentified); electronic, vibrational and rotational transitions are given in the last columns. In the list we have included many blended lines noted with a " + " sign.

\begin{tabular}{|c|c|c|c|c|c|}
\hline$\lambda(\AA)$ & $\begin{array}{l}\text { Peak } \\
\text { intensity }\end{array}$ & Species & $\begin{array}{l}\text { Electronic } \\
\text { transition }\end{array}$ & $\begin{array}{l}\text { Vibrational } \\
\text { transition }\end{array}$ & $\begin{array}{l}\text { Rotational } \\
\text { transition }\end{array}$ \\
\hline 6513.120 & 1.291 & Unid & & & \\
\hline 6513.468 & 2.262 & Unid & & & \\
\hline 6513.950 & 2.037 & $\mathrm{NH} 2$ & $X-X$ & $(0,12,0)-(0,0,0)$ & $110-221$ \\
\hline+ & & $\mathrm{NH} 2$ & $X-X$ & $(0,12,0)-(0,0,0)$ & $110-221$ \\
\hline 6514.140 & 1.110 & $\mathrm{C} 2$ & Swan & $4-7$ & $\mathrm{R} 2(6)$ \\
\hline 6514.779 & 1.171 & $\mathrm{C} 2$ & Swan & $4-7$ & $\mathrm{P} 2(27) \mathrm{P} 3(26) \mathrm{R} 3(5)$ \\
\hline 6514.920 & 1.248 & Unid & & & \\
\hline 6515.145 & 1.162 & $\mathrm{C} 2$ & Swan & $4-7$ & R1( 6) \\
\hline 6517.271 & 1.120 & Unid & & & \\
\hline 6517.469 & 2.239 & $\mathrm{NH} 2$ & $\mathrm{X}-\mathrm{X}$ & $(0,12,0)-(0,0,0)$ & $111-220$ \\
\hline 6517.730 & 1.110 & $\mathrm{NH} 2$ & $X-X$ & $(0,12,0)-(0,0,0)$ & $111-220$ \\
\hline 6518.377 & 1.759 & $\mathrm{NH} 2$ & $X-X$ & $(0,12,0)-(0,0,0)$ & $111-220$ \\
\hline 6519.489 & 1.707 & $\mathrm{NH} 2$ & $X-X$ & $(0,12,0)-(0,0,0)$ & $211-322$ \\
\hline
\end{tabular}

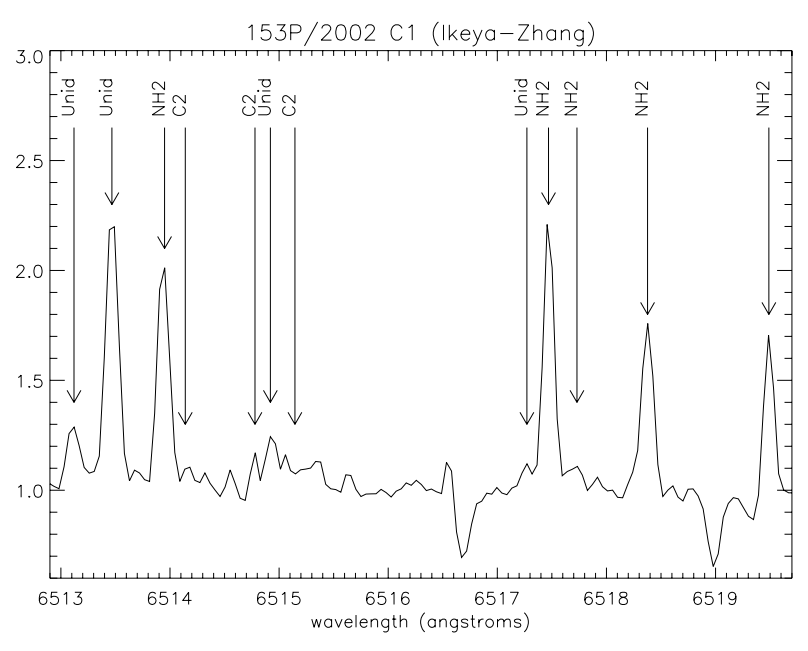

Fig. 2. Detail of order 39 with lines identifications. The label "Unid" means that the line is not identified.

because they are many and we know that $\mathrm{H}_{2} \mathrm{O}^{+}$can be found also in a spectrum not taken in the tail.

Furthermore we have found some atomic lines to be oxygen, that we have used to determine the ratio between the green and red emission (Capria et al. 2005), and the sodium D-lines, confirming their presence in this comet (Fig. 3).

In our catalog, 1862 lines are "Unidentified", some of which very strong. This is the most intriguing part of the catalog: while many of the lines are probably transitions from known species not listed in the laboratory catalogues, lines from minor unknown species could be hidden in this forest. Further laboratory work and more complete catalogs of measured lines are necessary. We have found in our spectrum several unknown lines not present in the catalog by Cochran \& Cochran (2002). Due to the fact that we are working with only one spectrum we choose to include in our tables only the lines whose identification is sure enough, and for the moment these lines are not listed. They will be the subject of further studies. We are also planning to collect laboratory measured lines of the molecules of interest, and make comparisons between all the comet spectra we have acquired.

Thus, we have analyzed high resolution visible spectrum of comet 153P/Ikeya-Zhang and listed 8468 emission lines. 6606 lines were attributed to a species, while 1862 unidentified.
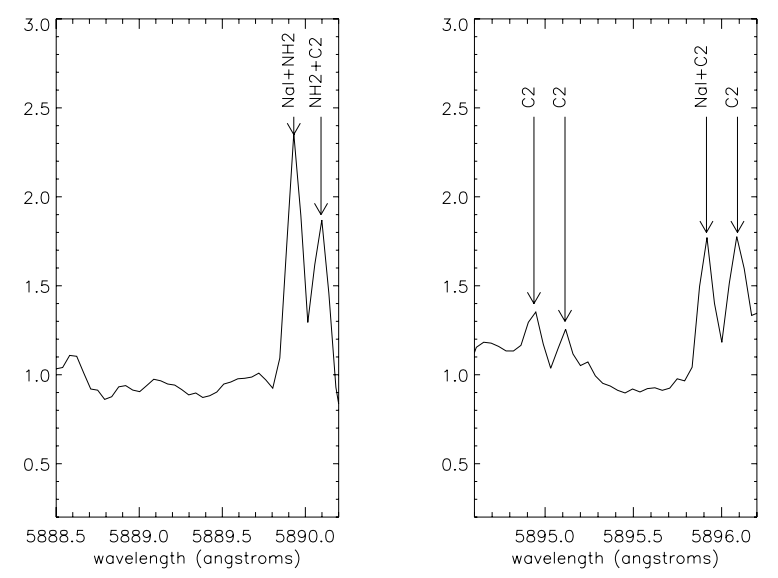

Fig. 3. Identification of the sodium D-lines from the order 30 .

The identification was based on the catalog published by Cochran \& Cochran (2002) and was done by wavelength coincidence, the spectra of the comet $122 \mathrm{P} / \mathrm{de}$ Vico are remarkably similar to our spectrum, so the context has been extensively used to confirm our interpretations.

Acknowledgements. Based on observations made with the Italian Telescopio Nazionale Galileo (TNG) operated on the island of La Palma by the Fundación Galileo Galilei of the INAF (Istituto Nazionale di Astrofisica) at the Spanish Observatorio del Roque de los Muchachos of the Istituto de Astrofisica de Canarias.

\section{References}

Arpigny, C. 1995, in Laboratory and Astronomical High Resolution Spectra, ed. A. J. Sauval, R. Blomme, \& N. Grevesse, ASP Conf. Ser., 81, 362

Brown, M. E., Bouchez, A. H., Spinrad, H., \& Johns-Krull, C. M. 1996, AJ, 112, 1197

Capria, M. T., Cremonese, G., Bhardwaj, A., \& De Sanctis, M. C. 2005, A\&A, 442,1121

Cochran, A. L., \& Cochran, W. D. 2002, Icarus, 157, 297

Ikeya, K., \& Zhang, D. 2002, IAUC, 7812

Kawakita, H., \& Watanabe, J. 2002, ApJ, 574, L183

Kawakita, H., Watanabe, J., Fuse, T., et al. 2004, ApJ, 601, 1152

Marsden, B. G., \& Nakano, S. 2002, IAUC, 7843

Osterbrock, D. E., Fulbright, J. P., Martel, A. R., Keane, M. J., \& Trager, S. C. 1996, PASP, 108, 277

Zhang, H. W., Zhao, G., \& Hu, J. Y. 2001, A\&A, 367, 1049 